\title{
Spatial Resolution of Autoradiograms of Rod-shaped Organisms
}

\author{
By ARTHUR L. KOCH \\ Department of Biology, Indiana University, Bloomington, Indiana 47405, U.S.A.
}

(Received 22 June 1981; revised 19 March 1982)

\begin{abstract}
Isotope-containing rod-shaped bacteria approximate a line-segment source for purposes of autoradiography. This is because the width is small and bacteria are usually cylindrically symmetric. For this reason published work has only classified the silver grains longitudinally, independent of the transverse position. While the theoretical integral distribution from a variety of geometric shapes has been calculated, the line-segment has been overlooked because long narrow objects well separated from other sources occur infrequently in eukaryotic biology. This oversight is corrected here. In addition, a simple program for line-segments and ways to combine distributions from separate line-segment sources is presented. Some typical distributions are depicted. The program is used elsewhere in the analysis of wall growth patterns of bacteria.
\end{abstract}

Autoradiography of tritium-labelled specimens has inherently low resolving power because the track of the $\beta^{-}$particle emanating from the radioactive decay has a length comparable to the dimension of most bacteria. The resolution can be improved by the method of Caro (1962), which uses a very thin layer of emulsion that is then viewed with an electron microscope. The resolution is better because electrons not travelling directly from the source towards the emulsion have a proportionately lesser chance of causing a latent image when the emulsion is thin.

Salpeter et al. (1969) experimentally measured the resolution of several variants of this electron microscope technique. They expressed their results in terms of HD, the half distance, i.e. the experimental distance from a line source in which half of the grains are located. They also provided graphs of computer calculations for a variety of labelling patterns to be expected for differently-shaped labelled objects.

This paper treats one important geometrical case that they did not consider, i.e. a uniformly labelled line-segment source. This case is applicable to the autoradiography of rod-shaped bacteria. For cylindrically symmetric bacteria, it is necessary and practical in most cases to consider only the distribution of grains along the length of the organism and place no significance on the perpendicular distance from the cell axis. For this reason, in all the autoradiographic studies of rod-shaped bacteria in the literature, the data have always been analysed by tallying the grains according to the distance from some reference line perpendicular to the axis of a cell, e.g. the line going through the midline or a developing cell division site. In this way information about the distance transverse to the axis of the cell in which the disintegration occurred has been omitted from further consideration by the longitudinal grouping. This procedure is, of course, very reasonable in many cases. For example, when the labelled molecule in the cell is peptidoglycan or phospholipid, the tritium atoms are almost certainly cylindrically distributed. Other cellular components, such as DNA, appear to be partially cylindrically distributed. However, even if the component is not cylindrically distributed, the grains in the final tally are cylindrically distributed because averages are 


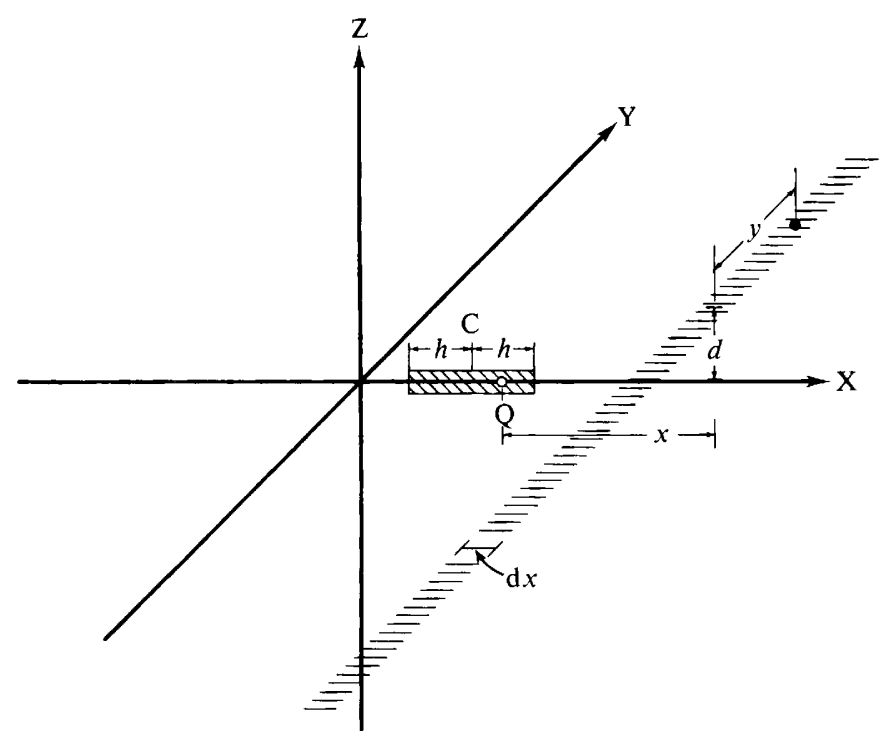

Fig. 1. Geometric relationship between a point or line-segment radioactive source and a perpendicular swath of thin emulsion in a plane above the plane of the source. This figure shows the symbols and geometric relationship between a point source, $\mathrm{Q}$, or a line source stretching between a distance $h$ on the plus and on the minus side of a point, $\mathrm{C}$, and either a point in the emulsion (designated by the solid circle) or an infinite strip (indicated by the hatched area) stretching from $\mathrm{Y}=-\infty$ to $\mathrm{Y}=+\infty$.

necessarily taken over many bacteria which have random rotational orientation on the electron micrograph.

Therefore, I present here the theoretical distributions of grains in small distance intervals along the length of the cell, but summed over all distance perpendicular to the long axis. For simplicity, I will give the derivation using the same symbols as defined by Salpeter et al. (1969). They used the symbol, $d$, as an approximation of HD (see below). Consider a point source of radioactivity lying at $\mathrm{Q}$ on the $\mathrm{X}$-axis (Fig. 1). Assume the layer of thin emulsion lies a distance $\mathrm{Z}=d$ above this but in the $\mathrm{X}-\mathrm{Y}$ plane. We wish to calculate the grains produced in the swath of emulsion at $\mathrm{X}=x$, where $x$ could be the coordinate of $\mathrm{Q}$, but could also be any place from $X=\infty$ to $X=-\infty$. The grains in the swath due to the radioactivity emanating from point $Q$ would be given by summing the radiation intensity at this $\mathrm{X}$ and $\mathrm{Z}(=d)$ location for all possible values of $Y$. This is expressed by:

$$
g_{\mathrm{p}}=K \int_{-\infty}^{+\infty} f_{\mathrm{p}}\left(x^{2}+y^{2}\right)^{1 / 2} \mathrm{~d} y
$$

where $g_{\mathrm{p}}$ stands for the grains due to a point source recorded in the narrow swath, $\mathrm{d} x$ wide, of emulsion parallel to the $\mathrm{Y}$-axis. $K$ is a constant containing efficiency factors and unit conversions. Following Salpeter et al. (1969), $f_{\mathrm{p}}$ is defined as the grains from a source point measured at a point in the film; it is the dose from a point source, Q, measured at a point $d$ above and at a distance away in that plane of $\left(x^{2}+y^{2}\right)^{1 / 2}$. They show that $f_{\mathrm{p}}$ is given to good approximation [modified from their equation (5)] by:

$$
f_{\mathrm{p}}=1 /\left\{1+\left(x^{2}+y^{2}\right) / d^{2}\right\}^{3 / 2}
$$

On substitution and integration, one obtains:

$$
g_{\mathrm{p}}=2 K d^{3} /\left(d^{2}+x^{2}\right)
$$

A second integration allows us to take into account all the grains coming from different portions of the line-segment source. If the line source is centred at $x=\mathrm{C}$ and if the half-length of 

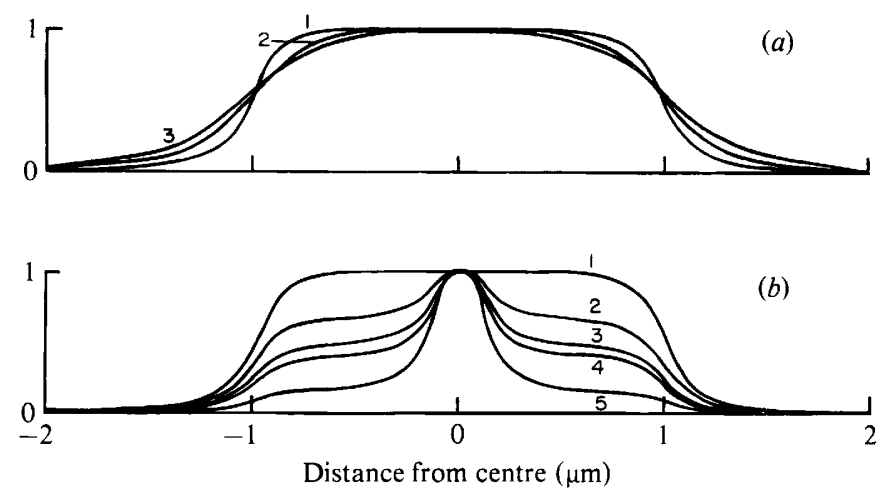

Fig. 2. Distribution of grains around a $2 \mu \mathrm{m}$ line-segment source. All cases were calculated for a line source stretching from $-1 \mu \mathrm{m}$ to $+1 \mu \mathrm{m}$. The ordinate is the total of grains summed perpendicularly from the line source. In this and Fig. 3 the curves have been normalized so that the maximum is 1 .

(a) Effect of variation in the resolution (as measured by $d$, which approximates HD): curve $1, d=100$ $\mathrm{nm}$; curve $2, d=200 \mathrm{~nm}$; curve $3, d=300 \mathrm{~nm}$.

(b) Effect of a central septum: curve 1, no septum; curve 2, amount of septal material required to complete a single double-thickness cross-wall of radius $=0.4 \mu \mathrm{m}$; curve 3 , radius $=0.8 \mu \mathrm{m}$; curve 4 , radius $=1.2 \mu \mathrm{m}$; curve 5 , radius $=4 \mu \mathrm{m}$. The septum in all cases was assumed to be $0.2 \mu \mathrm{m}$ thick and $d$ was assumed to be $100 \mathrm{~nm}$ which is close to the best resolution obtainable.

the source is $h$, then the grains on a swath perpendicular to the $\mathrm{X}$-axis and parallel to the $\mathrm{Y}$-axis due to all the points on the line source will be given by:

or

$$
g_{1}=2 d^{3} K \int_{C-h}^{c+h}\left\{d^{2}+(\mathrm{X}-x)^{2}\right\}^{-1} \mathrm{~d} x
$$

$$
g_{1}=2 d^{2} K\left[\tan ^{-1}\{(\mathrm{X}-\mathrm{C}-h) / d\}-\tan ^{-1}\{(\mathrm{X}-\mathrm{C}+h) / d\}\right]
$$

This equation can serve as the basis for more realistic cases where $d$ and/or $K$ vary for disintegrations occurring in different points of the transverse cross-section of the organism. But a good first approximation is that $d$ and $K$ are constant (see below). This equation also can serve as a basis for calculation of the distribution of grains for non-uniform longitudinal distributions of radioactivity by numerically integrating this one more time to sum over a collection of linesegment sources. This can be done readily by considering combinations of superimposed line sources with different values of $\mathrm{C}$ and $h$. Usually two sources suffice, say, a diffuse growth component and a narrow growth zone component.

By way of example, I will consider distributions relevant to the study of bacterial cell wall formation. Figure $2(a)$ shows the distribution of grains around a uniform linear source $2 \mu \mathrm{m}$ long for various values of $d(100,200$ and $300 \mathrm{~nm})$. These curves would also apply to actual cells if the walls are uniformly thick and uniformly labelled and if the poles are hemispherical. This is because a hemispherical pole has the same area, $2 \pi r^{2}$, and the same length, $r$, as would the corresponding open-ended cylinder. Figure $2(b)$ shows the distribution around a uniform source $2 \mu \mathrm{m}$ long on which a narrow central region is superimposed. This simulates the effect corresponding to a developing septum in Gram-positive organisms or a constrictive division in Gram-negative organisms. Curve 5 would correspond to the effect of pulse-labelling a Grampositive rod that was forming a septum and also adding in a diffuse manner to the cylindrical part.

Figure 3(a) shows the distribution of grains around the halves of an admixture of linear sources of slightly varying length. It is assumed that the grains for each line-segment are tallied by distance measured from the centre of each. A broader distribution of sources leads to a more gradual decrease in the frequency of grains compared with the case where all sources have the same length. Figure $3(b)$ would apply to long-term uniformly labelled cells; it shows the effect of 

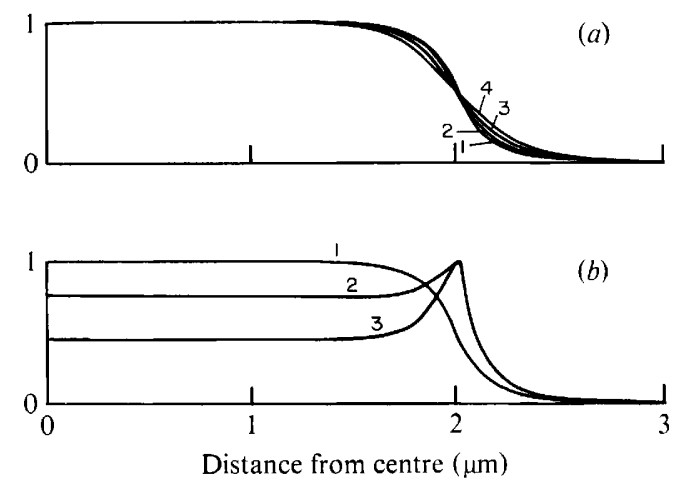

Fig. 3. (a) Effect of variability of half-length: curve 1, line source of $2 \mu \mathrm{m}$ half-length; curve 2, mixture of equal parts of line sources of $1.9,2 \cdot 0$ and $2.1 \mu \mathrm{m}$ half-length; curve 3 , mixture of equal parts of line sources of $1 \cdot 8,1 \cdot 9,2 \cdot 0,2 \cdot 1$ and $2 \cdot 2 \mu \mathrm{m}$ half-length; curve 4 , mixture of equal parts of line sources of $1 \cdot 7$, $1 \cdot 8,1 \cdot 9,2 \cdot 0,2 \cdot 1,2 \cdot 2$ and $2 \cdot 3 \mu \mathrm{m}$ half-length.

(b) Effect of pole surfaces (half-length of cell, $2 \mu \mathrm{m}$; uniform thickness of wall; uniform labelling; $d=$ $100 \mathrm{~nm}$ ): curve 1, a hemispherical pole; curve 2, flat (anthrax-like) pole; curve 3, flat pole for a cell with twice the diameter as for curve 2 .

the end-walls for the case of flat (anthrax-like) cells of radius $0.5 \mu \mathrm{m}$ (curve 2) and radius $1.0 \mu \mathrm{m}$ (curve 3). A flat end-wall produces a terminal bump whose magnitude depends on the radius of the cell. As noted above, a hemispherical pole leads to the same distribution (curve 1) as an openended cylinder. Poles more flattened than hemispheres, but not completely flat, would yield curves of intermediate character between curve 1 and those like curves 2 or 3 .

Evidently, for uniformly labelled cells the presence of extra wall in flattened poles of the cell, over and above the amount that a hemispherical pole has, tends to give a peak of grains above the poles (Fig. $3 b$ ). On the other hand, when classified from the centre, the variation in lengths within a size classification tends to make the concentration of grains decrease more gradually with distance from the centre of the cell (Fig. $3 a$ ). Together, these effects tend to compensate. The first effect can be isolated from the second by measuring the grains from the nearest pole and not from the cell centre as has usually been done. Since the pole shape can be measured, the theoretical curve for the case where radioactive atoms are distributed uniformly in all portions of the wall can be computed by summing appropriate line-segment sources. But the major question for which autoradiography has been used is, 'Where is new material added to the wall?' Consequently, it is the distribution after pulse-labelling that is important. For such studies as well it is important to make measurements of distance from the poles and from the centre (or site of the next cell division). Such an approach is presented in Koch et al. (1982) and Koch (1982, 1983).

The calculations are approximate in that the equation for $f_{\mathrm{p}}$ is derived from a geometric argument and is not fully realistic from the point of view of the paths that actual electrons of various energies make as they leave the helium recoil nucleus. Therefore, $d$ is only approximately HD. Salpeter et al. (1969) gave an empirical formula to improve the fit. I (Koch, 1965) used a more realistic formula from the point of view of radiation physics. However, the differences of the distributions for swath integrals from cylindrical sources of the size of bacteria (i.e. of a length long compared with HD) are negligible as shown by more complex simulations (calculations not shown). Consequently, the simplified form of $f_{\mathrm{p}}$ used here is adequate. An advantage of this simplification is that the calculations can be made on an advanced programable pocket calculator. Those presented here were performed and graphed on a Hewlett Packard HP 4IC pocket calculator with its peripheral printer. The program will be supplied by the author to those who wish it. A program can be easily set up with any computer and expanded to yield the distribution of line-segment sources that best fit the observed grain count histogram. 
A comparison of the procedure developed here and another one developed recently in several laboratories (Blackett \& Parry, 1977; Downs \& Williams, 1978; Salpeter et al., 1978) is appropriate. For the analysis of sectioned eukaryotic material, integral expressions are not usually applicable since organelles of complex shapes can occur in a variety of sizes and orientations and distances between them. With slight variation, these three groups of workers propose carrying out a sophisticated, but hand-operated, Monte Carlo integration. Thus, one assumes a distribution of disintegration events on a particular autoradiogram. For example, one might assume a uniform and constant distribution within a particular kind of organelle. Then one calculates a position of a resulting grain based on random or systematic chosen directions and located at distances that are drawn randomly from the distances of travel of the isotope under the conditions in question. One then sums the 'hypothetical grains' at a location of interest from all assumed radioactive regions. The distribution of hypothetical grains is then compared with the observed distribution. If a good fit is not obtained, the assumed distribution of radioactivity is changed and the calculation repeated. Such calculations are very timeconsuming, but are necessary in those cases. In our case, the more accurate and simple integration procedure works well and would be approximated by the hypothetical grain procedures only with an infinite number of draws for the case of sources arranged along a straight line-segment.

A final point must be made. The procedure as used above assumed that all disintegrations from all regions of the cell had the same half distance, $d$, and the same efficiency of producing a grain, $K$. These are appropriate assumptions if the sections or organisms (if whole mounted) are thin and the emulsion is uniform in thickness. But calculations are still possible if the efficiency is different for disintegrations emanating from different regions; they can be made if it is known or assumed just how the efficiency and half distance change. In principle, $d$ would be different for disintegrations coming from the top or bottom wall of a whole mounted cell; $K$ would be different if the film is not of uniform thickness. For example, if the emulsion is thinner when overlying the organism, disintegrations emanating from the sides of a cell might traverse a thicker layer of emulsion than disintegrations occurring at the top or bottom of the cell. If the variation in efficiency is known as a function of location, then an accurate, though more complex, calculation can be done for rod-shaped organisms based on the equation developed here.

Work in my laboratory is supported by the National Science Foundation under Grant PCM 79-1141.

\section{REFERENCES}

BlacketT, N. M. \& Parry, D. M. (1977). A simplified method of "hypothetical grain" analysis of electron microscope autoradiograms. Journal of Histochemistry and Cytochemistry 25, 206-214.

CARo, L. G. (1962). High resolution autoradiography. II. The problem of resolution. Journal of Cell Biology 15, 189-199.

Downs, A. \& Williams, M. A. (1978). An iterative approach to the analysis of EM autoradiographs. I. The method. Journal of Microscopy 114, 143-156.

KocH, A. L. (1965). A distributional basis for the variation in killing efficiencies by different tritiated compounds incorporated into Escherichia coli. Radiation Research 24, 398-411.

$\mathrm{KoCH}$, A. L. (1982). On the growth and form of Escherichia coli. Journal of General Microbiology 128, 2527-2539.
KoCH, A. L. (1983). The surface stress theory of microbial morphogenesis. Advances in Microbial Physiology (in the Press).

Koch, A. L., Verwer, R. W. H. \& Nanninga, N. (1982). Incorporation of diaminopimelic acid into the old poles of Escherichia coli. Journal of General Microbiology (in the Press).

Salpeter, M. M., BachmanN, L. \& Salpeter, E. E. (1969). Resolution in electron microscope radioautography. Journal of Cell Biology 41, 1-20.

SAlPeter, M. M., McHenry, F. A. \& SAlPeter, E. E. (1978). Resolution in electron microscope autoradiography: IV. Application to analysis of autoradiographs. Journal of Cell Biology 76, 127-145. 